\title{
Multiple-Antenna-Aided OFDM Employing Genetic-Algorithm-Assisted Minimum Bit Error Rate Multiuser Detection
}

\author{
Mohamad Y. Alias, Sheng Chen, and Lajos Hanzo, Fellow, IEEE
}

\begin{abstract}
The family of minimum bit error rate (MBER) multiuser detectors (MUD) is capable of outperforming the classic minimum mean-squared error (MMSE) MUD in terms of the achievable bit-error rate (BER) owing to directly minimizing the BER cost function. In this paper, we will invoke genetic algorithms (GAs) for finding the optimum weight vectors of the MBER MUD in the context of multiple-antenna-aided multiuser orthogonal frequency division multiplexing (OFDM) . We will also show that the MBER MUD is capable of supporting more users than the number of receiver antennas available, while outperforming the MMSE MUD.
\end{abstract}

Index Terms-Algorithm, genetic, minimum bit error rate, multiuser detector, orthogonal frequency division multiplexing (OFDM), space division multiple-access (SDMA).

\section{INTRODUCTION}

I $\mathrm{N}$ AN EFFORT to increase the achievable system capacity of an orthogonal frequency division multiplexing (OFDM) system, antenna arrays can be employed for supporting multiple users in a space division multiple-access (SDMA) communications scenario [1], [2]. A variety of linear multiuser detectors (MUDs) have been proposed for performing the separation of OFDM users based on their unique, user-specific, spatial signature, provided that their channel impulse response (CIR) was accurately estimated [1], [2]. The most popular SDMA-receiver design strategy is constituted by the minimum mean-squared error (MMSE) MUD, which was extensively characterized in [1] along with numerous other MUDs.

However, as recognized in [3]-[5] in a code division multipleaccess (CDMA) context, a better strategy is to choose the linear detector's coefficients so as to directly minimize the error-probability or bit-error ratio (BER), rather than the meansquared error (MSE). This is because minimizing the MSE does not necessarily guarantee that the BER of the system is also minimized. The family of detectors that directly minimizes the BER is referred to as the minimum bit-error rate (MBER) detector class [3]-[6]. The MBER criterion has been successfully applied in the linear equalization of binary signaling [4], for decision feedback equalization (DFE) [7], and in linear MIMO receivers [6]. It has also been shown that the MBER detector can be used effectively for linear multiuser detection in CDMA systems [3], [5].

Manuscript received April 14, 2004; revised August 26, 2004. The review of this paper was coordinated by Prof. T. Lok.

The authors are with School of Electronics and Computer Science, University of Southampton, SO17 1BJ, U.K. (e-mail: 1h@ecs.soton.ac.uk).

Digital Object Identifier 10.1109/TVT.2005.851303
In a CDMA system, each user is separated by a unique userspecific spreading code. However, given a certain bandwidth, the effective data rate is determined by the ratio of the chip rate and the spreading factor. As opposed to a CDMA system, an SDMA system differentiates each user by the associated unique, user-specific CIR encountered at the receiver antenna. Therefore each user will be able to transmit in a multiuser environment without reducing the data rate by the spreading factor (SF). Hence, the combination of SDMA and OFDM is attractive, especially for high-rate transmission. In a rather simplistic, but conceptually appealing interpretation, one could argue that the unique user-specific CIR plays the role of a user-specific CDMA signature, without reducing the data rate according to the SF of a hypothetical CDMA system. In this analogy the CIR signatures are not orthogonal to each other, but this is not a serious impediment, because even orthogonal spreading codes become nonorthogonal upon convolution by the CIR. However, owing to the nonorthogonal nature of the CIRs an efficient multiuser receiver is required for separating the users.

In [8] we have provided a formula for the exact MBER MUD weight calculation of an uplink SDMA OFDM system. We have also shown that the MBER MUD may significantly outperform the MMSE MUD in terms of the achievable BER in a twouser OFDM scenario. The novelty of this contribution is that we propose a new genetic algorithm (GA)-assisted MBER MUDaided OFDM system, as a design alternative to the simplified conjugate gradient (CG) algorithm of [8]. Our complexity comparisons show that the GA-based MBER MUD is capable of achieving the MBER performance at a lower complexity than that of the CG MBER MUD. We will also show that, unlike the MMSE MUD, the MBER MUD has the capability of supporting more users than the number of receivers at the base station.

The outline of the paper is as follows. Section II describes our system model, and Section III proposes a new GA-aided SDMA MUD. Section IV characterizes the achievable performance, whereas Section V quantifies the attainable complexity reduction. Finally, Section VI offers our conclusions.

\section{SYSTEM MODEL}

\section{A. Space Division Multiple Access}

Fig. 1 portrays the antenna array-aided uplink transmission scenario considered. In this figure, each of the $L$ simultaneous users is equipped with a single transmission antenna, whereas the base station's receiver capitalizes on a $P$-element antenna front end. The set of complex signals, $x_{p}[n, k], p \in 1, \ldots, P$ 


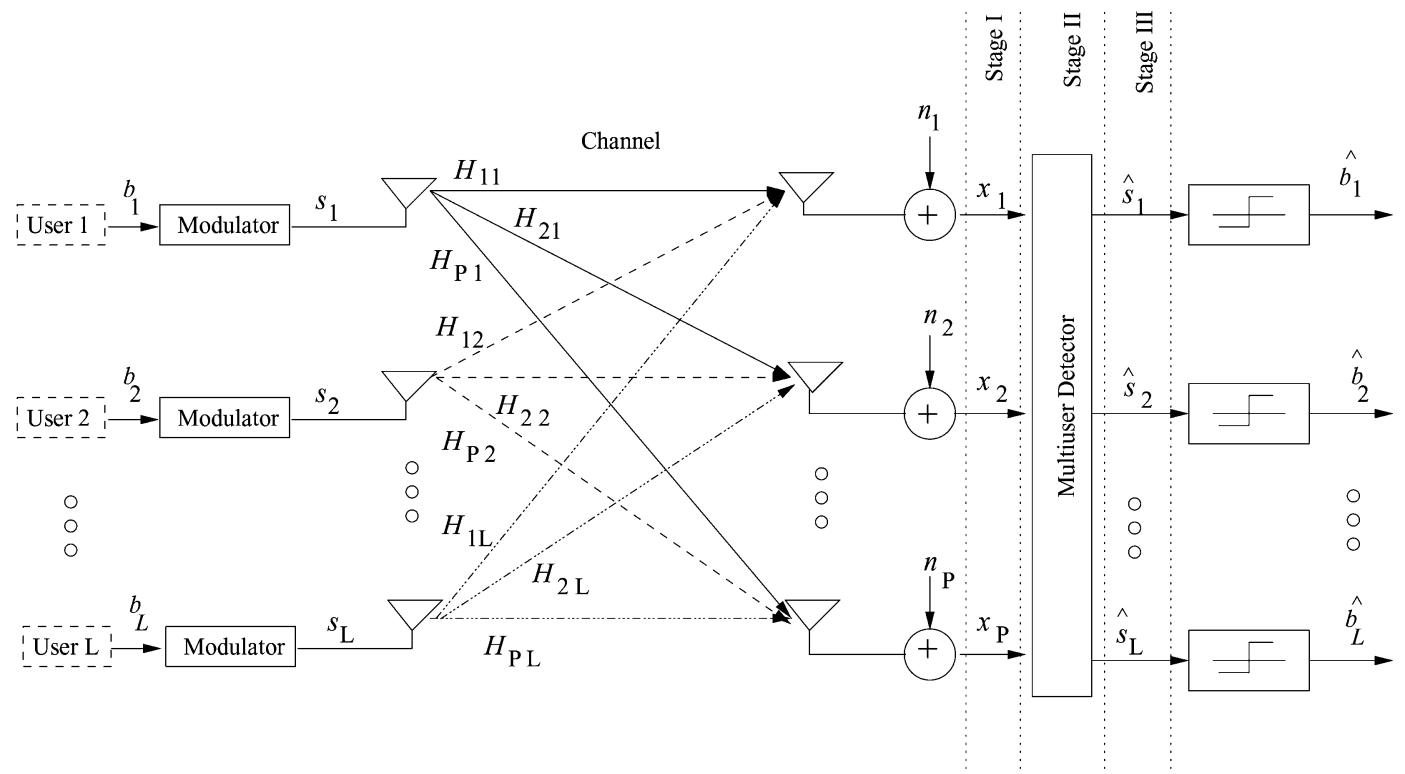

Fig. 1. Schematic of an antenna-array-aided OFDM uplink scenario, where each of the $L$ users is equipped with a single transmit antenna and the BS's receiver is assisted by a $P$-element antenna front-end.

received by the $P$-element antenna array in the $k$ th subcarrier of the $n$th OFDM symbol is constituted by the superposition of the independently faded signals associated with the $L$ users sharing the same space-frequency resource [1]. The received signal was corrupted by the Gaussian noise at the array elements. The indices $[n, k]$ have been omitted for notational convenience during our forthcoming discourse, yielding [1]

$$
\mathbf{x}=\mathbf{H s}+\mathbf{n}=\overline{\mathbf{x}}+\mathbf{n}
$$

where $\mathbf{x}$ is the $(P \times 1)$-dimensional vector of the received signals, $\mathbf{s}$ is the $(L \times 1)$-dimensional vector of transmitted signals, $\mathbf{n}$ is the $(P \times 1)$-dimensional noise vector, and $\overline{\mathbf{x}}$ represents the noiseless component of $\mathbf{x}$. The complex data signal, $s_{l}$, transmitted by the $l$ th user, $l \in 1, \ldots, L$ and the additive white Gaussian noise (AWGN) process, $n_{p}$, at any antenna array element $p, p \in 1, \ldots, P$ are assumed to exhibit a zero mean and a variance of $\sigma_{l}^{2}$ and $2 \sigma_{n}^{2}$ for the data signal and AWGN process, respectively. The frequency domain channel transfer function (FDCHTF) matrix $\mathbf{H}$ of dimension $P \times L$ is constituted by the set of channel transfer function vectors of the $L$ users, each of which describes the FDCHTF between the single-transmitter antenna associated with a particular user $l$ and the reception array elements $p \in 1, \ldots, P$. The FDCHTFs, $H_{p l}$ of the different array elements $p \in 1, \ldots, P$ for users $l \in 1, \ldots, L$ are independent, stationary, and complex Gaussian distributed processes with zero-mean and unit variance. The users are assumed to be at a sufficiently high distance from each other, namely at a distance of more than a typical spacing of $10 \lambda$ [9], so that the transmitted signals of the different antennas experience independent fading, when they reach the receiver. By contrast, in the scenario, where the users are in each other's proximity, methods like beam forming [10] exploiting the difference in the angle of arrival among the users may be applied for differentiating the users.
The estimate $\hat{\mathbf{s}}$ of the transmitted signal vector $\mathbf{s}$ of the $L$ simultaneous users is generated by the MUD upon linearly combining the signals received by the $P$ different antenna elements at the BS with the aid of the array weight matrix $\mathbf{W}$, resulting in

$$
\hat{\mathbf{s}}=\mathbf{W}^{H} \mathbf{x}
$$

By substituting $\mathbf{x}$ from (1) into (2) and considering the $l$ th user's associated vector component, we will arrive at

$$
\begin{aligned}
\hat{s}_{l} & =\mathbf{w}_{l}^{H} \mathbf{x}=\mathbf{w}_{l}^{H} \mathbf{H} \mathbf{s}+\mathbf{w}_{l}^{H} \mathbf{n} \\
& =\bar{s}_{l}+\mathbf{w}_{l}^{H} \mathbf{n}
\end{aligned}
$$

where the weight vector $\mathbf{w}_{l}$ is the $l$ th column of the weight matrix W. At the current state-of-the-art, the most popular MUD strategy is the MMSE design, where $\mathbf{w}_{l}$ is chosen as the unique vector minimizing the MSE expressed as MSE $=E\left[\left|\hat{s}_{l}-s_{l}\right|^{2}\right]$, namely as [1]

$$
\mathbf{w}_{l(\mathrm{MMSE})}=\left(\mathbf{H H}^{H}+2 \sigma_{n}^{2} \mathbf{I}\right)^{-1} \mathbf{H}_{l}
$$

where $\mathbf{H}_{l}$ is the $l$ th column of the FDCHTF matrix $\mathbf{H}$.

\section{B. Error Probability of a BPSK System}

In this treatise, the terms BER and probability of error $P_{E}$ are used interchangeably. In a BPSK system we have $b_{l} \in\{+1,-1\}$, the BER encountered at the output of the SDMA MUD characterized by the combiner weight vector $\mathbf{w}_{l}$ of user $l$ may be expressed as [5]

$$
\begin{aligned}
P\left(\mathbf{w}_{l}\right) & =\operatorname{Pr}\left[\operatorname{sgn}\left(b_{l}\right) \cdot \Re\left\{\bar{s}_{l}\left(\mathbf{w}_{l}\right)\right\}<0\right], \\
& =\operatorname{Pr}\left[z_{l}<0\right]
\end{aligned}
$$

where $z_{l}$ is the signed decision variable given by

$$
z_{l}=\operatorname{sgn}\left(b_{l}\right) \cdot \Re\left\{\bar{s}_{l}\left(\mathbf{w}_{l}\right)\right\}
$$


while, as before, $b_{l}$ represents the transmitted bit of user $l, \bar{s}_{l}$ is the noiseless signal at the output of the MUD related to the $l$ th user, and $\Re\{x\}$ is the real part of the complex-valued $x$.

The probability density function (PDF) of the decision variable $z_{l}$ is constituted by a mixture of the Gaussian distributions associated with each possible combination of the transmitted data symbols of all users. Under the assumption that all the noise-free signal states are equiprobable, the PDF of $z_{l}$ is given by [5]

$$
p_{z_{l}}\left(z_{l} ; \mathbf{w}_{l}\right)=\frac{1}{N_{b} \sqrt{2 \pi} \sigma_{n} \sqrt{\mathbf{w}_{l}^{H} \mathbf{w}_{l}}} \sum_{j=1}^{N_{b}} e^{\left(-\frac{\left(z_{l}-\operatorname{sgn}\left(b_{l}^{(j)}\right) \bar{s}_{l}^{(j)}\right)^{2}}{2 \sigma_{n}^{2} \mathbf{w}_{l}^{H} \mathbf{w}_{l}}\right)}
$$

where $N_{b}$ is the number of equiprobable combinations of the binary vectors of the $L$ users, i.e., we have $N_{b}=2^{L}$. Furthermore, $\bar{s}_{l}^{(j)}, j \in 1, \ldots, N_{b}$, denotes the real part of the noiseless signal at the output of the MUD related to the $l$ th user, whereas $b_{l}^{(j)}, j \in 1, \ldots, N_{b}$, is the transmitted bit of user $l$.

The erroneous decision events are associated with the area under the PDF curve in the interval $(-\infty, 0)$, which is quantified as

$$
P_{E}\left(\mathbf{w}_{l}\right)=\int_{-\infty}^{0} p_{z_{l}}\left(z_{l} ; \mathbf{w}_{l}\right) d z_{l} .
$$

Upon using the integration by substitution technique and introducing the shorthand of

$$
y_{j}=\frac{\left(z_{l}-\operatorname{sgn}\left(b_{l}^{(j)}\right) \bar{s}_{l}^{(j)}\right)}{\sigma_{n} \sqrt{\mathbf{w}_{l}^{H} \mathbf{w}_{l}}}
$$

the probability of error in (8) becomes

$$
\begin{aligned}
P_{E}\left(\mathbf{w}_{l}\right) & =\frac{1}{N_{b} \sqrt{2 \pi}} \sum_{j=1}^{N_{b}} \int_{-\infty}^{c_{j}\left(\mathbf{w}_{l}\right)} \exp \left(-\frac{\left(y_{j}\right)^{2}}{2}\right) d y_{j} \\
& =\frac{1}{N_{b}} \sum_{j=1}^{N_{b}} Q\left[c_{j}\left(\mathbf{w}_{l}\right)\right]
\end{aligned}
$$

where $c_{j}\left(\mathbf{w}_{l}\right)$ is given by

$$
c_{j}\left(\mathbf{w}_{l}\right)=\frac{\operatorname{sgn}\left(b_{l}^{(j)}\right) \cdot \bar{s}_{l}^{(j)}}{\sigma_{n} \sqrt{\mathbf{w}_{l}^{H} \mathbf{w}}}=\frac{\operatorname{sgn}\left(b_{l}^{(j)}\right) \cdot \Re\left\{\mathbf{w}_{l}^{H} \overline{\mathbf{x}}_{j}\right\}}{\sigma_{n} \sqrt{\mathbf{w}_{l}^{H} \mathbf{w}}}
$$

and $\overline{\mathbf{x}}_{j}, j \in 1, \ldots, N_{b}$ constitutes a possible value of $\overline{\mathbf{x}}$ defined in the context of (1).

\section{Exact MBER Multiuser Detection}

In our initial discourse we assumed the explicit knowledge of the FDCHTF matrix $\mathbf{H}$ defined in (1). However, in practice $\mathbf{H}$ has to be determined on the basis of the channel-impaired noisy value of $\mathbf{x}$ and, hence, several adaptive techniques have been proposed in [4], [5] to this effect. The FDCHTF can also be estimated by using various channel estimation methods as outlined in great detail in [1], which will hence not be investigated

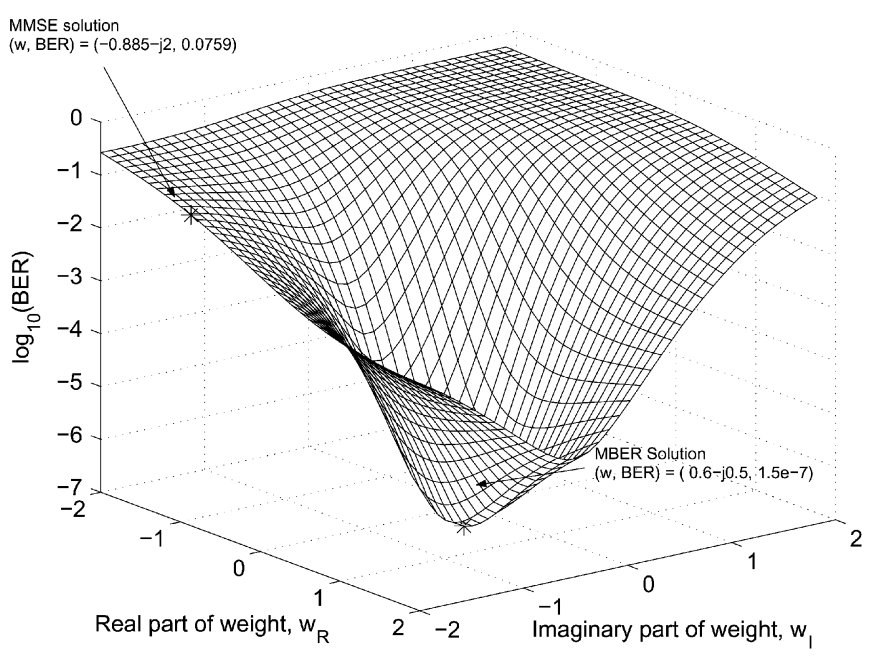

Fig. 2. An example of the BER cost function surface for subcarrier 62 in the $P=2, L=2$ OFDM SDMA system employing 128-subcarrier in the symbolinvariant dispersive Gaussian channel given in Table I at SNR $=10 \mathrm{~dB}$.

in this treatise. The MBER solution is defined as [5]

$$
\mathbf{w}_{l(\mathrm{MBER})}=\arg \min _{\mathbf{w}_{l}} P_{E}\left(\mathbf{w}_{l}\right) .
$$

However, the complex, irregular shape of the BER cost function, for example, as shown in Fig. 2, prevents us from deriving a closed-form solution for the MBER MUD weights. Therefore, in practice an iterative strategy based on the steepest descent gradient method may be used for finding the MBER solution [5]. According to this method, the linear SDMA MUD's weight vector $\mathbf{w}_{l}$ is iteratively updated, commencing for example from the MMSE weights, until the specific SDMA MUD weight vector that exhibits the lowest BER is arrived at. In each step, the weight vector is updated according to a specific step size $\mu$ in the vectorial direction in which the BER cost function decreases most rapidly, namely in the direction opposite to the gradient of the BER cost function, which is given by

$$
\begin{gathered}
\nabla_{\mathbf{w}_{l}} P_{E}\left(\mathbf{w}_{l}\right)=\frac{1}{N_{b} \sqrt{2 \pi} \sigma_{n}}\left(\frac{\mathbf{w}_{l} \mathbf{w}_{l}^{H}-\mathbf{w}_{l}^{H} \mathbf{w}_{l} \mathbf{I}}{\left(\mathbf{w}_{l}^{H} \mathbf{w}_{l}\right)^{\frac{3}{2}}}\right) \\
\cdot \sum_{j=1}^{N_{b}} e^{\left(-\frac{\left.\bar{s}_{l}^{(j)}\right)^{2}}{2 \sigma_{n}^{2} \mathbf{w}_{l}^{H} \mathbf{w}_{l}}\right)} \cdot \operatorname{sgn}\left(b_{l}^{(j)}\right) \cdot \overline{\mathbf{x}}_{j}
\end{gathered}
$$

where $\bar{s}_{l}^{(j)}=\Re\left\{\mathbf{w}_{l}^{H} \overline{\mathbf{x}}_{j}\right\}$.

The BER is independent of the magnitude of the MUD's weight vector [8], and hence the knowledge of the orientation of the detector's weight vector is sufficient for defining the decision boundary of the linear MBER OFDM/SDMA detector. Therefore, the MBER detector has an infinite number of MUD weight solutions, although with the aid of appropriate weightvector normalization, it is possible to reduce the infinite number of MBER solutions to a single solution.

In [8], we employed the simplified conjugate gradient (CG) algorithm for arriving at the minimum solution of the BER cost function, and the step size $\mu$ is fixed for every iteration. 


\section{Genetic Algorithm}

Even though the MBER detector of [8] is capable of maintaining a good performance, the convergence of the algorithm is sensitive to the choice of the algorithm's parameters. For example, the choice of the initial condition for the MBER MUD is critical for the solution to converge to the minimum of the BER surface seen in Fig. 2. In [8], the MMSE SDMA MUD weight solution has been used for initializing the CG algorithm-based MUD, which is also exemplified in Fig. 2. However, this choice of initial conditions does not necessarily guide the algorithm's convergence to the required MBER solution, if the BER surface exhibits local minima, although this is not the case in Fig. 2. Another parameter that affects the performance of the MBER detector is the step size $\mu$ used for updating the array weights in the direction opposite to the BER gradient. The choice of this step size must be based on a compromise, since a step-size that is too high might not allow convergence to the minimum BER point, whereas the opposite scenario will require a high number of iterations for attaining convergence to the MBER solution. An attractive method that might be able to assist the MBER MUD in circumventing the above-mentioned problems is constituted by the family of genetic algorithms [11], which were extensively used in various CDMA and MC-CDMA MUD problems in [12].

\section{A. Overview of GAs}

The philosophy of GA-based search methods is reminiscent of the rules of evolution and survival in nature, where a group of the fittest individuals in a population will survive. These individuals will then "mate" and "mutate" for the sake of developing a new set of individuals for the next generation. GAs were proposed by John Holland at the University of Michigan in the early 1970s [13]. His original target was to study the adaptive process of a natural system and later to adapt this natural system in the context of an artificial system software. Holland's research was further developed by one of his students, Goldberg [11]. Since then, GAs have been employed in numerous applications, such as in machine learning [14]-[16] and in modeling adaptive processes [17], [18], but the most frequent application of GAs may be found in the domain of function optimization [11], [13], [19]. GAs have also been successfully applied in CDMA [12], [20]-[27], beam forming [28], and space-time block coding (STBC) [29] aided multiuser detection scenarios.

GAs are different from traditional optimization algorithms, because they do not directly attempt to optimize the desired decision variable [11]. Instead, they encode the decision variables to be optimized, such as, for example, the SDMA MUD's weight vectors, into finite-length strings or GA individuals, which are then optimized. In the case of SDMA-MUDs, both the real and imaginary part of a complex-valued weight has to be represented by a single GA string to create an individual. A GA does not commence its optimization process from a single point in the search space, but rather from an entire set of individuals, which form the initial population. In other words, GAs may be invoked in robust global search and optimization procedures that do not require the knowledge of the objective function's derivatives or

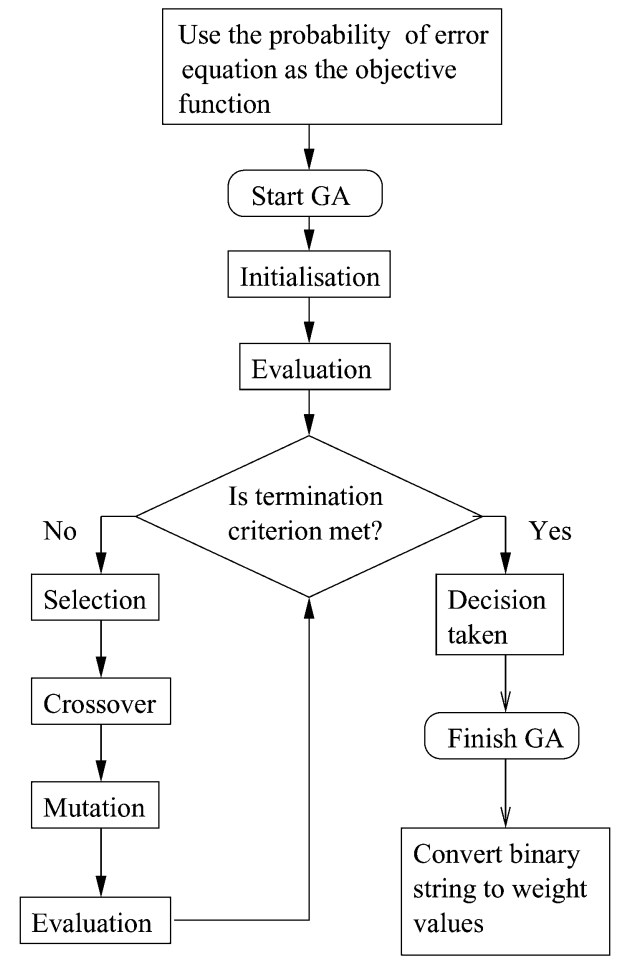

Fig. 3. Flowchart for the probability error optimization using GA.

any gradient-related information concerning the search space. Hence, nondifferentiable functions and functions with multiple local minima, like the BER surface of the SDMA-MUD, represent classes of problems, where GAs can be efficiently applied. For further details on the origin of GAs and their application, readers are referred to the impressive compilation of ideas in [11], [13], [30]. The most detailed portrayal of the GA-aided CDMA MUDs may be found in [12].

\section{B. Employing GAs in the MBER MUD-Aided SDMA OFDM System}

In this contribution, a GA is used for finding the best SDMAMUD weight vectors that will minimize the probability of error in (10). Fig. 3 shows the flow chart of a GA invoked for the optimization of the MBER MUD's weights. The basic approach of a GA system is quite simple. First, the probability of error is used as the objective function to be solved by the GA. To begin the GA process, an initial population consisting of $Y$ number of so-called individuals is created in the "Initialization" block, where $Y$ is known as the population size. Each individual represents a legitimate solution to the given optimization problem. An individual can be considered as a vector consisting of the decision variables to be optimized. Traditionally, the individuals in a GA population take the form of binary bit vectors, but they can also be represented by real values. In our specific problem, each individual in a population is represented by the two-dimensional binary arrays, which store the real and imaginary part of the SDMA-MUD's weight values. An example of the GA's individual is shown in Fig. 4. The initial population may be generated randomly, optionally including as one of the 


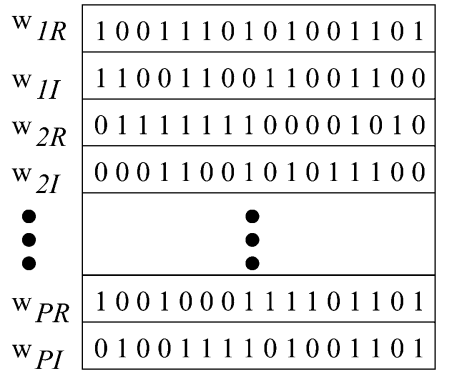

Fig. 4. An example of an individual in the GA system represented by the twodimensional binary arrays where $P$ is the number of antennas at the receiver, $R$ is the real part of the weight, and $I$ is the imaginary part of the weight.

individuals the MMSE solution for the sake of expediting the search as well as for reducing the complexity of the search.

Each individual in the population is evaluated according to the so-called fitness value associated with it. This fitness value is calculated by substituting the individual into the objective function in the "Evaluation" block seen in Fig. 3. Following the fitness evaluation process, the termination criterion will be checked, which may be either a certain convergence accuracy, a certain number of generations, or various other criteria. We opted for terminating the evaluations after $G$ number of generations. If the stopping criterion is not met, a group of the highest fitness individuals is selected for creating a generation in the "selection" block of Fig. 3. This group of individuals, referred to as parents, will be subjected to various genetically inspired operators, such as the so-called crossover and mutation, for the sake of creating new individuals. The fitness of these new individuals will then be reevaluated and the termination criterion is examined again.

This process will continue until the termination criterion is finally met. After this stage, the best individual having the highest fitness encountered will be chosen as the solution to the optimization problem.

\section{Simulation Results}

\section{A. Performance of a Four-User and Four-Receiver Antenna Scenario}

The parameters used in our simulations are outlined in Table I. The channel that we used in these investigations was the unfaded dispersive Gaussian channel, where the $z$-domain transfer function associated with the CIR of all four users and four antennas are summarized in Table II. Since the different users experienced different CIR taps at each receiver antennas, we also had unique FDCHTFs for the various users. As a starting point, we used binary-type genomes [11] to represent the individual GAs. Therefore, each real and imaginary part of each of the SDMA MUD weights is represented by a 16-bit binary string. The GA's termination criterion is constituted by the maximum affordable number of generations. After the termination of the optimization process, the best individual encountered is deemed to be the best MBER MUD weight solution, and hence it is converted to the corresponding real and imaginary values of the weight vectors. Our results derived for four different users employing the pa-
TABLE I

PARAMETERS FOR THE GA SIMULATIONS

\begin{tabular}{l|r}
\hline Parameter & Value or Type \\
\hline \hline OFDM & 128 \\
\hline Number of subcarriers & 32 \\
\hline Length of cyclic prefix & nonoverlapping \\
\hline GA & 100 \\
\hline GA type & 100 \\
\hline Population size & flip mutator \\
\hline Number of generations & 0.01 \\
\hline Mutation type & single-point crossover \\
\hline Probability of mutation & dispersive Gaussian channel [1] \\
\hline Crossover type & binary encoding and decoding \\
\hline Probability of crossover & roulette wheel \\
\hline Scaling & bigma truncation \\
\hline Genome type & binary string \\
\hline Initialization & uniform \\
\hline Comparison & on comparator \\
\hline Encoding/decoding & sich is specified for each user in Table II \\
\hline Selection & \\
\hline Elitism & \\
\hline Others & \\
\hline Receiver antennas & \\
\hline MBER detector algorithm & \\
\hline Channel impulse response & \\
\hline
\end{tabular}

rameters summarized in Table I are presented in Fig. 5. In these investigations we imposed the same complexity, as quantified explicitly in Section V for both the CG and GA aided MBER MUDs. The figure shows that the GA-aided MBER MUD and the CG MBER MUD perform similarly for the same complexity. We can also see from the figure that the MMSE MUD's BER performance is different for each user because of the different CIRs experienced at the base-station antennas. On the other hand, the results of both types of MBER MUDs differs only slightly for the different users, and the achievable BER of the different users is close to that of the single-user singleantenna scenario recorded for a nondispersive AWGN channel. We have also derived the average of the achievable BER of all the users determined for the CIRs summarized in Table II and the corresponding results are portrayed in Fig. 6.

\section{B. Performance of the Four-Antenna Scenario Versus the Number of Users}

So far, we have been supporting the same number of users as the number of receiver antennas in our simulations. By contrast, 


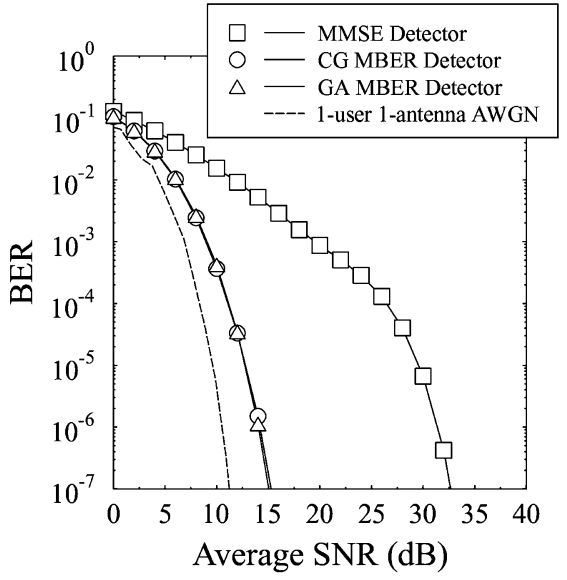

(a)

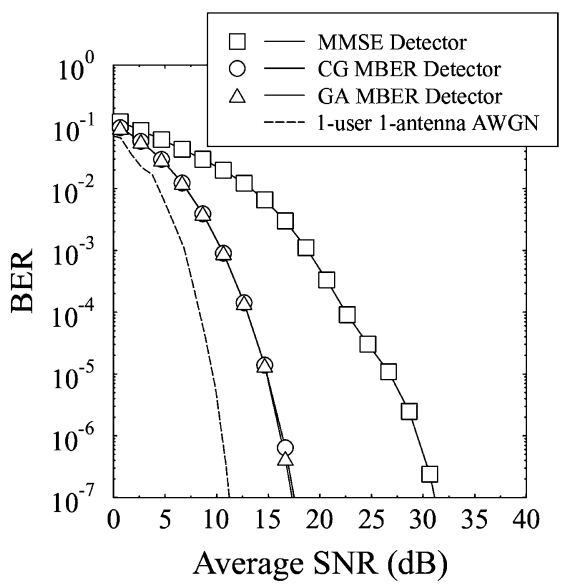

(c)

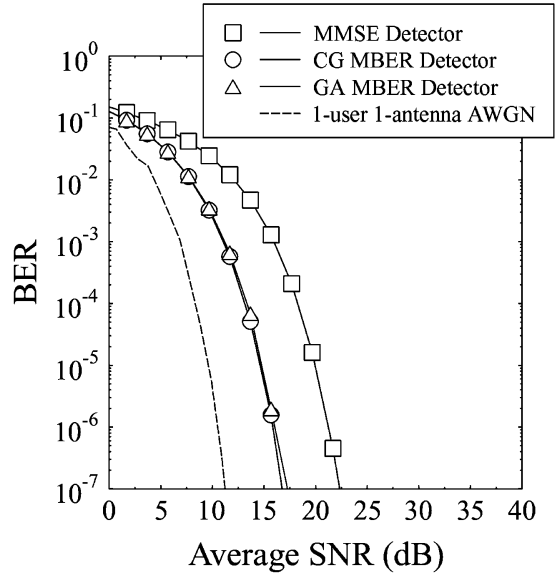

(b)

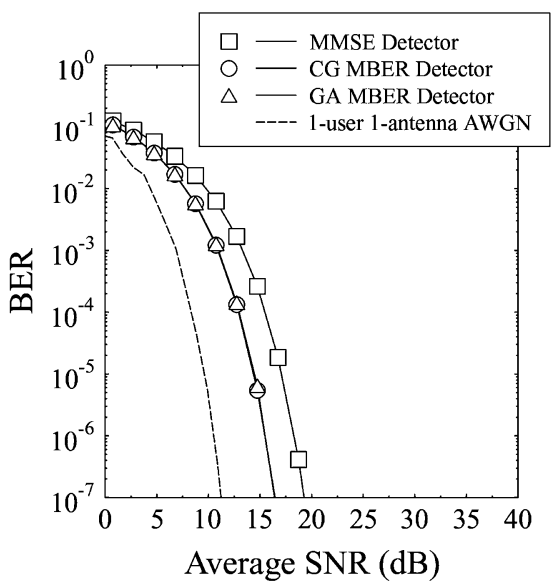

(d)

Fig. 5. The BER performance of the four different users in an SDMA system employing four receiver antennas and 128-subcarrier OFDM for communicating over the OFDM symbol-invariant dispersive Gaussian channel given in Table II. The other parameters are summarized in Table I. (a) User 1. (b) User 2. (c) User 3. (d) User 4.

TABLE II

CIR OF THE DIFFERENT USERS AT THE DIFFERENT ANTENNAS FOR THE $P=4, L=4$ SYSTEM

\begin{tabular}{l|l|l}
\hline User & Antenna & CIR \\
\hline \hline \multirow{4}{*}{ User 1 } & Antenna 1 & $(-0.097-j 0.062)-(0.56+j 0.40) z^{-6}-(0.30-j 0.014) z^{-11}$ \\
\cline { 2 - 3 } & Antenna 2 & $(-0.50-j 0.51)-(0.116334+j 0.50) z^{-6}+(0.37-j 0.12) z^{-11}$ \\
\cline { 2 - 3 } & Antenna 3 & $(-0.13-j 0.20)+(0.19+j 0.31) z^{-6}+(0.21-j 0.16) z^{-11}$ \\
\cline { 2 - 3 } & Antenna 4 & $(-1.41+j 0.17)-(0.88-j 0.13) z^{-6}+(0.71+j 0.25) z^{-11}$ \\
\hline \multirow{4}{*}{ User 2 } & Antenna 1 & $(-0.24-j 0.32)+(0.24+j 0.18) z^{-6}+(0.25+j 0.38) z^{-11}$ \\
\cline { 2 - 3 } & Antenna 2 & $(0.48-j 0.27)-(0.16+j 0.27) z^{-6}-(0.041+j 0.24) z^{-11}$ \\
\cline { 2 - 3 } & Antenna 3 & $(0.84+j 0.57)+(0.10-j 0.15) z^{-6}+(0.61+j 0.018) z^{-11}$ \\
\cline { 2 - 3 } & Antenna 4 & $(-0.57-j 0.43)-(0.17+j 0.090) z^{-6}+(0.11+j 0.15) z^{-11}$ \\
\hline \multirow{4}{*}{ User 3 } & Antenna 1 & $(-0.79+j 1.43)+(0.082+j 0.35) z^{-6}+(0.16-j 0.59) z^{-11}$ \\
\cline { 2 - 3 } & Antenna 2 & $(0.53-j 0.52)+(0.37+j 0.11) z^{-6}+(0.29+j 0.29) z^{-11}$ \\
\cline { 2 - 3 } & Antenna 3 & $(0.76-j 0.070)-(0.032-j 0.62) z^{-6}+(0.43-j 0.11) z^{-11}$ \\
\cline { 2 - 3 } & Antenna 4 & $(0.85+j 0.104)-(0.068-j 0.42) z^{-6}+(0.080-j 0.46) z^{-11}$ \\
\hline \multirow{4}{*}{ User 4 } & Antenna 1 & $(1.92+j 0.12)-(0.10+j 0.60) z^{-6}-(0.17+j 0.0049) z^{-11}$ \\
\cline { 2 - 3 } & Antenna 2 & $(0.42-j 0.47)+(0.0095+j 0.097) z^{-6}-(0.21+j 0.23) z^{-11}$ \\
\cline { 2 - 3 } & Antenna 3 & $(-0.022+j 0.21)+(0.059-j 0.069) z^{-6}+(0.21-j 0.11) z^{-11}$ \\
\cline { 2 - 4 } & Antenna 4 & $(0.45-j 0.12)-(0.12-j 0.57) z^{-6}-(0.30+j 0.21) z^{-11}$ \\
\hline
\end{tabular}

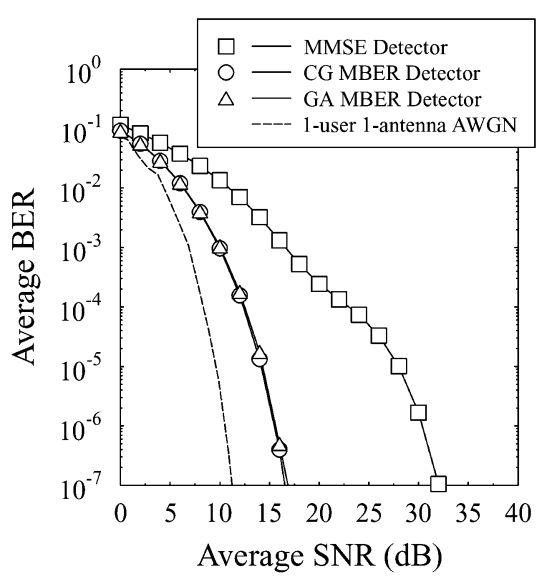

Fig. 6. The average BER performance of the four different users characterized in Fig. 5 and ecountering the CIRs of Table II in an SDMA system employing four receiver antennas and 128-subcarrier OFDM for communicating over the OFDM symbol-invariant dispersive Gaussian channel given in Table I.

Fig. 7 shows the BER results for user 1, when supporting a different number of users in the four receiver antenna scenario. 


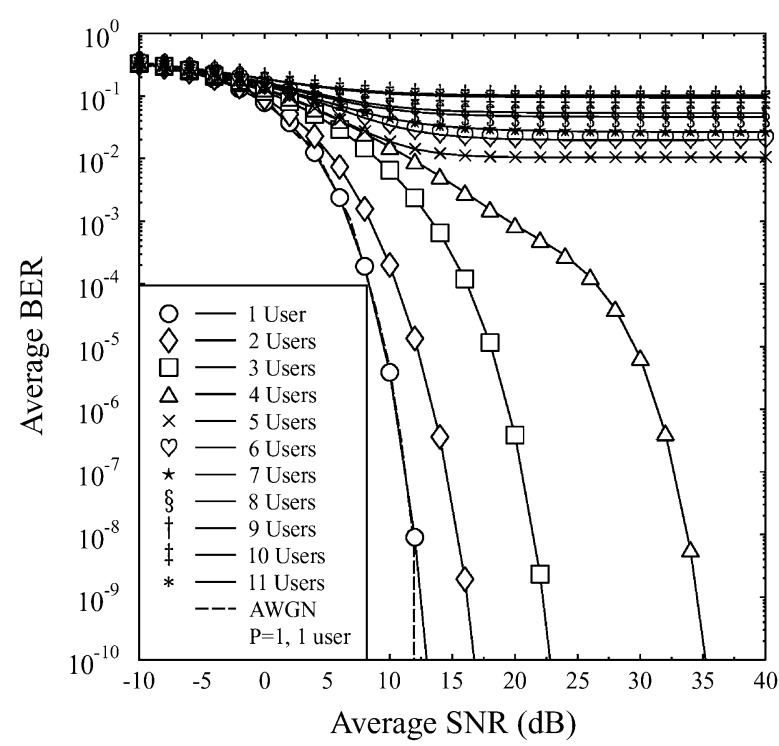

(a)

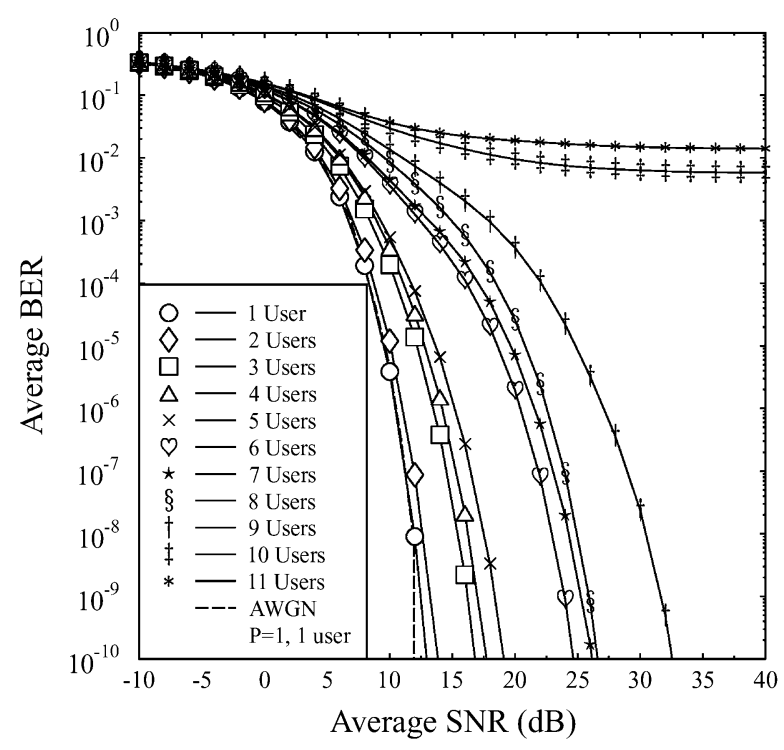

(b)

Fig. 7. The BER performance of user 1 employing both the MMSE and MBER MUD in an SDMA system equipped with four receiver antennas for different numbers of users employing 128-subcarrier OFDM communicating over the OFDM symbol-invariant dispersive Gaussian channel given in Table I. (a) MMSE MUD. (b) MBER MUD.

For the MBER MUD, we have used the GA-aided system introduced in Section III.B, employing the simulation parameters specified in Table I. We can see from the figure that as the number of users increases, the BER performance degrades owing to the increased multiuser interference imposed. In the absence of multiuser interference when only one user is communicating, both MUDs have similar BER curves. As expected, this is because no multiuser interference is inflicted and hence the MMSE MUD is also capable of minimizing the BER. However, the MMSE MUDs characterized in Fig. 7(a) can only support a maximum number of users that is equal to the number of receiver antennas, which is four in this case. Once the number of users exceeds the number of receiver antennas, the MMSE
MUD becomes incapable of differentiating the users, resulting in the high residual BER seen in Fig. 7(a). By contrast, we observed in Fig. 7(b) that the MBER MUD performs significantly better. Furthermore, by comparing Fig. 7(a) and (b) we may conclude that the MBER MUD is capable of supporting more users than the number of receiver antennas employed.

These performance trends may be more explicitly interpreted with the aid of Fig. 8, where the composition of user 1's noiseless received phasors are illustrated when transmitting $s_{1}=+1$ in the six-user scenario employing four receiver antennas. In this figure, the different processing stages are defined in Fig. 1. In short, stage I represents the composite multiuser received signals, $x_{1}, \ldots, x_{P}$ at the different antennas. The MUD stage II outputs the product of the Hermitian of the MUD's weight value, $w_{p}, p \in 1, \ldots, P$, of the individual antenna elements and the corresponding $x_{p}, p \in 1, \ldots, P$, value, i.e., $w^{H} x$. Finally, stage III represents the combination of the outputs of each antenna. Explicitly, the MMSE MUD attempts to minimize the Euclidean distance between the estimated received symbol $\hat{s}_{1}$ and the original transmitted symbol $s_{1}$. However, as can be observed at stage III of Fig. 8, some of the $\hat{s}_{1, \mathrm{MMSE}}$ points are either on the wrong half-plane of the BPSK phasor constellation or exactly on the decision boundary, at $y_{R}=0$. As a consequence, the associated residual BER experienced in the absence of noise can be calculated by taking into account the relative frequency of these points. On the other hand, since the MBER MUD is directly minimizing the BER, the MUD's weight values are adjusted for the sake of ensuring that the estimated phasors are as far away from the decision boundary as possible. Therefore, we can see from Fig. 8 that the estimated received phasors $\hat{s}_{1, \text { MBER }}$ are significantly further from the BPSK decision boundary of $y_{R}=0$, than for the MMSE MUD hence avoiding the MMSE-specific residual $\mathrm{BER}$.

\section{COMPLEXITY COMPARISON}

The advantage of using GAs for determining the MBER MUD's weight values compared with the CG method [8] is that the GA does not necessarily require a good initial MUD weight guess for exhibiting a rapid convergence. In this section, we will estimate the complexity of these two methods. In this paper, the complexity refers to the number objective function evaluations or the gradient evaluations for the GA and the $\mathrm{CG}$ methods, respectively.

The complexity of the CG algorithm is proportional to the number of iterations used for finding the MBER solution on the BER surface. In each iteration the gradient expression of (13) will have to be calculated and the SDMA-MUD weight values will be updated accordingly. Therefore the complexity of the CG method may be estimated as:

$$
\operatorname{Compl}\{\mathrm{CG}\} \simeq \operatorname{Maximum} \text { number of iterations. }
$$

On the other hand, if we used the maximum number of generations as the termination criterion in the GA, each generation of the population contains a certain number of individuals, thus the complexity of the GA-aided MUD is proportional to the product of the population size and to the number of generations used, 


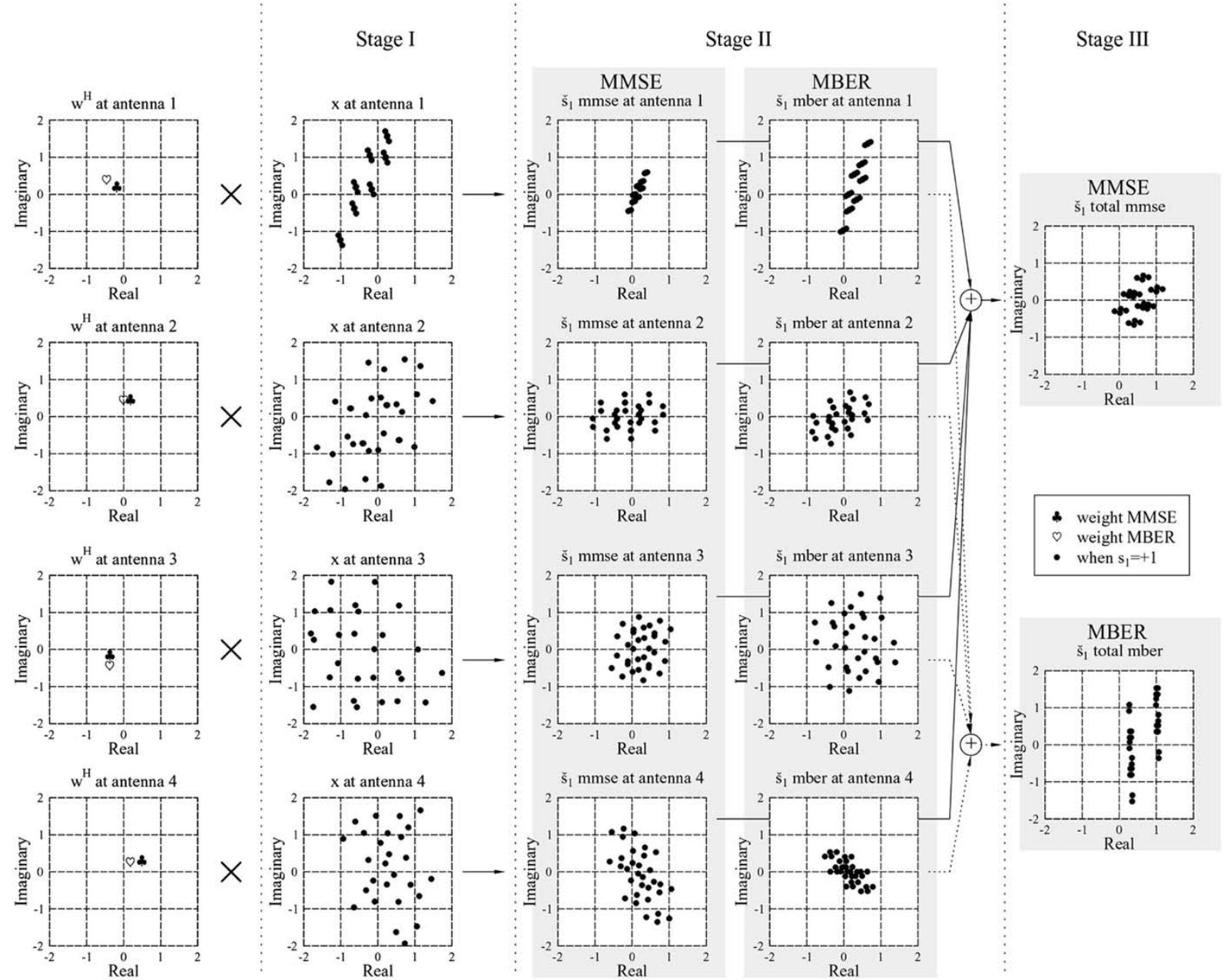

Fig. 8. An example composition of the probable noiseless received signal for user 1 for the case of $P=4, L=6$ when transmitting $s_{1}=+1$. The stages are as depicted in Fig. 1. Other parameters are given in Table I.

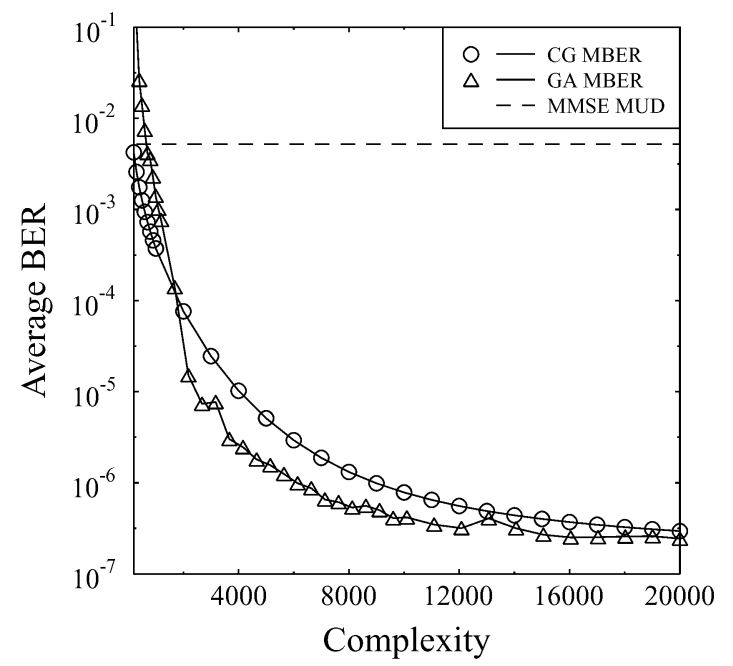

Fig. 9. The BER performance of user 1 versus complexity for the GA and CG MBER MUD invoked in the OFDM/SDMA system employing $\mathrm{P}=4, \mathrm{~L}=4$, and 128-subcarrier OFDM for communicating over the OFDM-symbol invariant dispersive Gaussian channel given in Table I at SNR $=15 \mathrm{~dB}$. The complexity calculations were described in Section V. which is given by

$$
\begin{aligned}
\operatorname{Compl}\{\mathrm{GA}\} & \simeq \text { Population size } \times \text { number of generations } \\
& =Y \times G .
\end{aligned}
$$

By using (14) and (15), we can compare the complexity of the two methods. Fig. 9 shows the probability of error for user 1 at $\mathrm{SNR}=15 \mathrm{~dB}$ for the $P=4$ and $L=4$ system configuration. We can see from the figure that the GA-aided SDMA-MUD will reach the minimum BER at a lower complexity compared to the CG method.

\section{CONCLUSION}

In this contribution, we have shown that GAs may be applied in the context of an SDMA OFDM system for determining the MBER MUD's weight vectors. The GA-aided system has an edge over the CG-based system, because it does not require an initial weight solution. Unlike the MMSE MUD, the MBER MUD is capable of supporting more users than the number of receiver antennas. It was also shown that the GA is capable 
of approaching the MBER solution at a lower complexity than the $\mathrm{CG}$ algorithm. Our future work will aim for finding more efficient adaptive weight optimization algorithms in the context of LDPC-coded SDMA OFDM systems.

\section{REFERENCES}

[1] L. Hanzo, M. Münster, B. J. Choi, and T. Keller, OFDM and MC-CDMA. West Sussex, Piscataway, NJ, England: Wiley, IEEE Press, 2003.

[2] P. Vandenameele, L. van Der Perre, and M. Engels, Space Division Multiple Access for Wireless Local Area Networks. Boston, MA: Kluwer, 2001.

[3] R. C. de Lamare and R. Sampaio-Neto, "Adaptive MBER decision feedback multiuser receivers in frequency selective fading channels," IEEE Commun. Lett., vol. 7, no. 2, pp. 73-75, Feb. 2003.

[4] C.-C. Yeh and J. R. Barry, "Adaptive minimum bit-error rate equalization for binary signalling," IEEE Trans. Commun., vol. 48, no. 7, pp. 12261235, Jul. 2000.

[5] S. Chen, A. K. Samingan, B. Mulgrew, and L. Hanzo, "Adaptive minimumBER linear multiuser detection for DS-CDMA signals in multipath channels," IEEE Trans. Signal Process., vol. 49, no. 6, pp. 1240-1247, Jun. 2001.

[6] D. Gesbert, "Robust linear MIMO receivers: A minimum error-rate approach," IEEE Trans. Signal Process., vol. 51, no. 11, pp. 2863-2871, Nov. 2003.

[7] B. Mulgrew and S. Chen, "Adaptive minimum-BER decision feedback equalizers for binary signalling," EURASIP Signal Process. J., vol. 81, no. 7, pp. 1479-1489, Jul. 2001.

[8] M. Y. Alias, A. K. Samingan, S. Chen, and L. Hanzo, "Multiple antenna aided OFDM employing minimum bit error rate multiuser detection," IEE Electron. Lett., vol. 39, no. 24, pp. 1769-1770, Nov. 27, 2003.

[9] J. H. Winters, "Smart antennas for wireless systems," IEEE Pers. Commun., vol. 5, no. 1, pp. 23-27, Feb. 1998.

[10] J. Blogh and L. Hanzo, $3 G$ Systems and Intelligent Networking. New York, Piscataway, NJ: Wiley, IEEE Press, 2002.

[11] D. E. Goldberg, Genetic Algorithms in Search, Optimization, and Machine Learning. Reading, MA: Addison-Wesley, 1989.

[12] L. Hanzo, L.-L. Yang, E.-L. Kuan, and K. Yen, Single- and Multi-Carrier DS-CDMA: Multi-User Detection, Space-Time Spreading, Synchronisation, Networking and Standards. West Sussex, England: Wiley, 2002.

[13] J. Holland, Adaptation in Natural and Artificial Systems. Ann Arbor, MI: University of Michigan Press, 1975.

[14] J. J. Grefenstette, Genetic Algorithms for Machine Learning: Kluwer, 1993.

[15] H. Adeli and S. L. Hung, Machine Learning: Neural Networks, Genetic Algorithms and Fuzzy Systems. New York: Wiley, 1994.

[16] S. K. Pal and P. P. Wong, Genetic Algorithms for Pattern Recognition. Boca Raton, FL: CRC Press, 1996.

[17] M. D. Vose, The Simple Genetic Algorithm: Foundations and Theory (Complex Adaptive Systems). Cambridge, MA: MIT Press, 1999.

[18] H. Dawid, Adaptive Learning by Genetic Algorithms: Analytical Results and Applications to Economic Models (Lecture Notes in Economics and Mathematical Systems). Berlin: Springer-Verlag, 1996.

[19] M. Gen and R. Cheng, Genetic Algorithms and Engineering Optimisation (Wiley Series in Engineering Design and Automation). New York: Wiley, 2000.

[20] M. J. Juntti, T. Schlosser, and J. O. Lilleberg, "Genetic algorithms for multiuser detection in synchronous CDMA," in Proc. of IEEE Symp. on Inf. Theory, Ulm, Germany, 29 Jun.-4 Jul. 1997, p. 492.

[21] X. F. Wang, W.-S. Lu, and A. Antoniou, "Genetic-algorithm-based multiuser detector for multiple-access communications," in Proc. IEEE Int. Symp. Circuits and Syst., vol. 4, Monterey, CA, 31 May-3 Jun. 1998, pp. 534-537.

[22] C. Ergun and K. Hacioglu, "Multiuser detection using a genetic algorithm in CDMA communications systems," IEEE Trans. Commun., vol. 48, no. 8, pp. 1374-1383, Aug. 2000.

[23] S. Abedi and R. Tafazolli, "A new CDMA multiuser detection technique using an evolutionary algorithm," in IEEE Proc. Commun., vol. 148, no. 6, Dec. 2001, pp. 393-399.

[24] M. G. Shayesteh, M. B. Menhaj, and B. G. Nobary, "A modified genetic algorithm for multiuser detection in DS/CDMA," IEICE Trans. Commun., vol. E86-B, no. 8, pp. 2377-2388, Aug. 2003.
[25] K. Yen and L. Hanzo, "Genetic algorithm assisted joint multiuser detection and fading channel estimation for synchronous cdma systems," IEEE $J$. Sel. Areas Commun., vol. 19, no. 6, pp. 985-998, Jun. 2001.

[26] — "Antenna-diversity-assisted genetic-algorithm-based multiuser detection schemes for synchronous CDMA systems," IEEE Trans. Commun., vol. 51, no. 3, pp. 366-370, Mar. 2003.

[27] S. X. Ng, K. Yen, and L. Hanzo, "TTCM assisted genetic-algorithm aided reduced-complexity multiuser detection," Electron. Lett., vol. 38, no. 14 pp. 722-724, Jul. 4, 2002.

[28] A. Wolfgang, N. N. Ahmad, S. Chen, and L. Hanzo, "Genetic algorithm assisted error probability optimisation for beam-forming," IEEE Electron. Lett., vol. 40, no. 5, pp. 320-322, Mar. 4, 2004.

[29] Y. Du and K. T. Chan, "Feasibility of applying genetic algorithms in space-time block coding multiuser detection systems," in Proc. IASTED Int. Conf. Wireless and Opt. Commun., vol. 3, Banff, Canada, Jul. 2-4, 2003, pp. 469-473.

[30] M. Mitchell, An Introduction to Genetic Algorithm. Cambridge, MA: MIT Press, 1996.

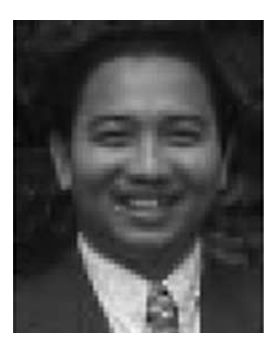

Mohamad Y. Alias obtained the Bachelor of Science in engineering (electrical engineering) degree from the University of Michigan, Ann Arbor, in May 1998. He then received the Ph.D. degree in December 2004 from the School of ECS, University of Southampton in the United Kingdom.

He is currently a Lecturer in the Faculty of Engineering with the Multimedia University in Malaysia. His research interests cover the field of OFDM, multiple antenna system, multiuser detection, and genetic algorithms in communications.

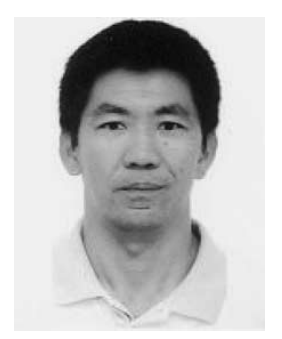

Sheng Chen (SM'97) received the B.Eng. degree in control engineering from the East China Petroleum Institute, Dongying, China, in 1982 and the Ph.D. degree in control engineering fromlahe City University, London, U.K., in 1986.

He joined the School of Electronics and Computer Science, University of Southampton, Southampton, U.K., in September 1999. He previously held research and academic appointments at the University of Sheffield, Sheffield, U.K., the University of Edinburgh, Edinburgh, U.K., and University of Portsmouth, Portsmouth, U.K. His recent research works include adaptive nonlinear signal processing, wireless communications, modeling and identification of nonlinear systems, neural network research, finite-precision digital controller design, evolutionary computation methods, and optimization. He has published over 200 research papers.

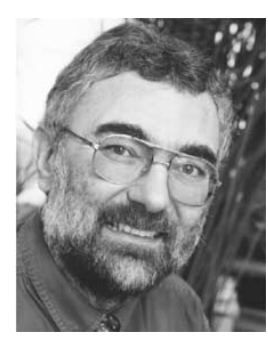

Lajos Hanzo (M'91-SM'92-F'04) received the M.S. degree in electronics in 1976 and the Ph.D. degree in 1983. In 2004 he was awarded the D.Sc. degree by the University Southampton, U.K.

During his 28-year career in telecommunications he has held various research and academic posts in Hungary, Germany, and the U.K. Since 1986 he has been with the Department of Electronics and Computer Science, University of Southampton, U.K., where he holds the chair in telecommunications. He has coauthored 11 books on mobile radio communications, published more than 500 research papers, organized and chaired conference sessions, presented overview lectures, and been awarded a number of distinctions. Currently he is managing an academic research team, working on a range of research projects in the field of wireless multimedia communications sponsored by industry, the Engineering and Physical Sciences Research Council (EPSRC) U.K., the European IST Programme, and the Mobile Virtual Centre of Excellence (VCE), U.K. He is an enthusiastic supporter of industrial and academic liaison and he offers a range of industrial courses. For further information on research in progress and associated publications, please refer to www-mobile.ecs.soton.ac.uk.

Dr. Hanzo is an IEEE Distinguished Lecturer of both the Communications Society and the Vehicular Technology Society and a Fellow of both the Royal Academy of Engineering (F.R.Eng.) and IEE. 